\title{
Improving the Quality of the Ion Beam Exiting a Quadrupole Ion Guide
}

\author{
Vadym D. Berkout and Vladimir M. Doroshenko \\ MassTech, Inc., Columbia, Maryland, USA
}

The effect of fringing fields on the divergence of the ion beam exiting an RF quadrupole ion guide was studied using a computer simulation. It was shown that reducing the strength of the $\mathrm{RF}$ field towards the ion guide exit reduces ion beam divergence. Further improvement was demonstrated when creating a DC gradient towards the exit. The results of the numerical simulation were verified experimentally using a time-of-flight (TOF) mass analyzer with orthogonal acceleration. Decreasing the ion beam divergence resulted in considerably improved mass resolution of the instrument. (J Am Soc Mass Spectrom 2006, 17, 335-340) (C) 2006 American Society for Mass Spectrometry

$\mathrm{T}$ The use of radiofrequency (RF) multipole ion guides for the efficient transport of ions produced at the atmospheric pressure into a mass analyzer operating under high vacuum is a common practice in mass spectrometry. A detailed description of the operation of RF-only ion guides was presented by Gerlich [1]. The ion guides employed in mass spectrometry are generally quadrupoles, hexapoles, or octopoles, and have found particular utility in regions where neutral gas pressure is high enough $\left(10^{-3}-10^{-2}\right.$ torr $)$ that multiple collisions take place with gas molecules. Radially confining effective potential well, generated in RF-only multipoles, significantly reduces losses and also cools ions translationally [2]. The benefits of using ion guides for efficient transmission of ions, generated by electrospray ionization (ESI), into a quadrupole ion trap were demonstrated by Stafford et al. [3]. The cooling of ions is especially beneficial in the case of a time-of-flight (TOF) mass analyzer with orthogonal acceleration (oa), where the energy spread of the ion beam exiting an RF ion guide irreversibly affects the resolution and sensitivity of TOF mass spectrometer [4].

In oa-TOF instruments, ions after leaving the RF ion guide are reaccelerated in the axial direction to the necessary energy and focused by electrostatic optics to make the beam divergence properties close to a parallel beam. The resulting ion beam is accelerated in the orthogonal $\left(\mathrm{y}^{-}\right)$direction by applying an extraction voltage pulse to the parallel acceleration plates. An electrostatic mirror can correct the energy spread resulting from ion initial spatial spread by compressing the ion packet when it approaches the detector [5]. It was pointed out [6] that the effect of the initial velocity spread in the $y$-direction on resolution of oa-TOF mass

Published online January 30, 2006

Address reprint requests to Dr. V. D. Berkout, MassTech, Inc., 6992 Columbia Gateway Dr., Columbia, MD 21046, USA. E-mail: vberkout@apmaldi.com spectrometers is probably the largest. The ion moving opposite to the time-of-flight direction will turn around after applying the extraction pulse and will get to the same starting plane with the velocity directed in the time-of-flight direction with some delay referred to as a turn-around time. The ion that had originally the same velocity directed in the time-of-flight direction will be ahead in time scale. The contribution in the peak width $\Delta t$ due to this effect is determined by [7]:

$$
\Delta t=\frac{2 m \mathrm{v}_{\mathrm{y}}}{z e E}
$$

where $\mathrm{v}_{\mathrm{y}}$ is the velocity spread in y direction, $m$ is the mass, $z e$ is the ion charge, and $E$ is the strength of the electric field during the extraction.

It is well known that fringe fields at the entrance and exit of quadrupole mass filters play an important role $[8,9]$. We suggest that the same phenomenon is taking place at the RF ion guide exit, thus increasing the energy spread of the ion beam leaving the ion guide. Here, we seek to optimize the quality of the ion beam entering the TOF mass analyzer by reducing the strength of the RF field towards the ion guide exit. We present SIMION 7.0 modeling to show the effect of fringe fields on ion beam divergence and propose methods to reduce it along with experimental demonstration on an oa-TOF mass spectrometer.

\section{Experimental}

\section{TOF Mass Spectrometer}

The TOF mass spectrometer with orthogonal acceleration and an ESI ion source is shown schematically in Figure 1. It consists of an electrospray ion source, atmospheric pressure interface, octopole ion guide, a system of quadrupole ion guides and a TOF mass analyzer. 
The atmospheric pressure (AP) interface consists of a heated capillary $(0.4 \mathrm{~mm}$ i.d.) and a quadrupole ion guide $(6.35 \mathrm{~mm}$ rod diameter). The pressure in the AP interface ( $\sim 1$ torr) was maintained by a $30 \mathrm{~m}^{3} / \mathrm{h}$ roughing pump. The octopole ion guide $(3.2 \mathrm{~mm}$ rod diameter) was operated at $\sim 0.1$ torr pressure. Both, the quadrupole and octopole were driven by an RF generator, built in-house, according to the design described in [10]. A capacitive divider allowed the application of different RF amplitudes to quadrupole and octopole ion guides, respectively.

A section of the quadrupole ion guides $(8 \mathrm{~mm}$ rod diameter) was separated from the octopole by a $2 \mathrm{~mm}$ diameter aperture. The quadrupoles were driven by a SRS (Sunnyvale, CA) model DS340 sine-wave signal generator coupled through an ENI (Rochester, NY) model 240L broadband RF power amplifier which exhibits flat gain over a wide range of operating frequencies. An RF coupling transformer, built in-house, gave an output voltage 0 to $500 \mathrm{~V}_{0-p}$ (zero-to-peak and pole to ground voltage) in the frequency range of $100 \mathrm{kHz}$ to 5 $\mathrm{MHz}$. The transformer also provided the required $180^{\circ}$ phase difference between the rod pairs. The quadrupole rods were offset to some DC potential, applied through decoupling capacitors. The DC voltages applied to the lens and aperture plate, as well as to the quadrupole, are adjusted to obtain optimum ion transmission. A schematic of the last segmented quadrupole is shown in Figure 2.

The DC divider consisted of a $110 \mathrm{k} \Omega$ resistor chain. The DC line was isolated from the RF one by $5 \mathrm{M} \Omega$ resistors. When $\mathrm{DC}_{\text {in }}$ and $\mathrm{DC}_{\text {out }}$ were connected to the same voltage all voltages on the quadrupole sections were the same. When $\mathrm{DC}_{\text {out }}$ was connected to ground, a DC gradient was created. A similar divider consisting of $220 \mathrm{pF}$ capacitors allowed changing the RF voltages applied to the individual rod sections. The value of 220 $\mathrm{pF}$ is much larger than rod capacitance thus causing minimal RF voltage variation on the quadrupole sec-

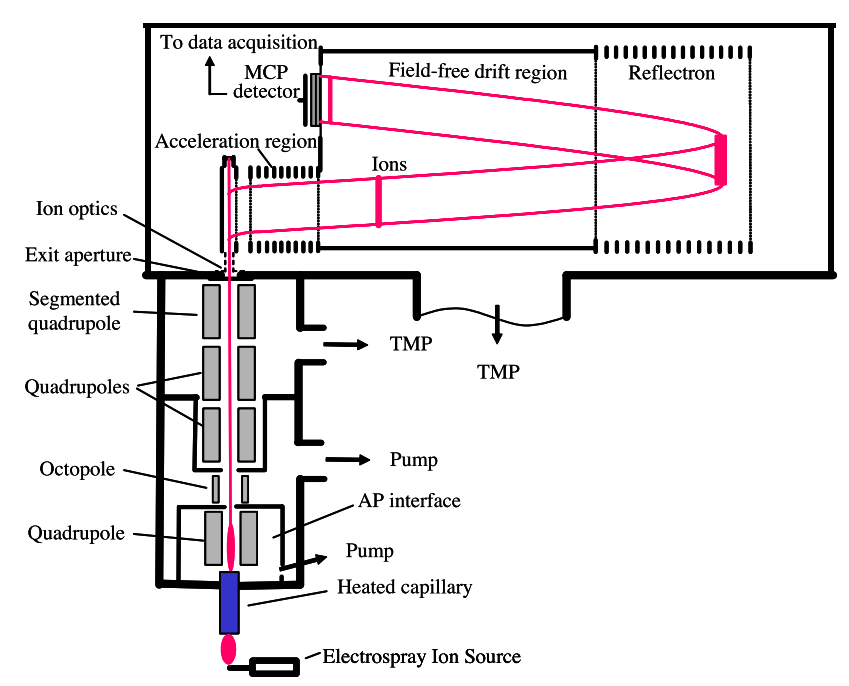

Figure 1. Schematic view of oa-TOF mass spectrometer.

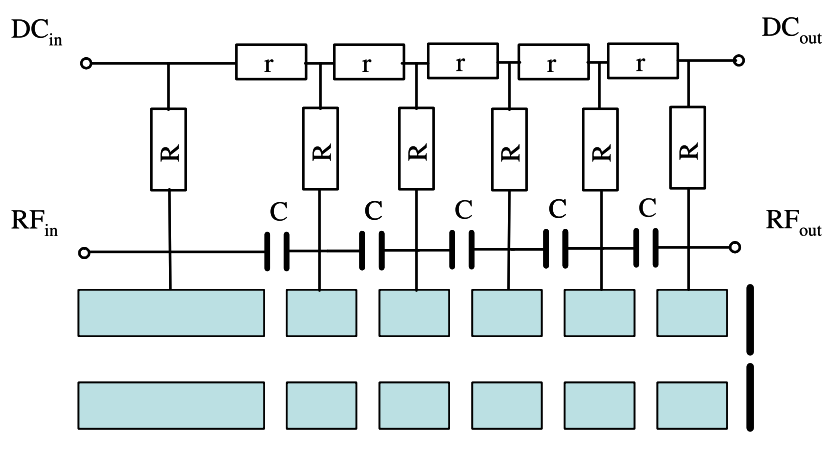

Figure 2. General schematic of the segmented quadrupole.

tions (less than $5 \%$ ) when the same RF voltage is applied to both ends. The length of the 1-st section was $25 \mathrm{~mm}$ and the next 5 sections were $9.5 \mathrm{~mm}$ long. Sections were separated by $1 \mathrm{~mm}$ thick PEEK washers. The pressure in the region with the segmented quadrupole was about $10^{-3}$ torr and was maintained by Edwards (Wilmington, MA) model EXT70 70 1/s turbomolecular pump.

After passing through the final aperture $(1 \mathrm{~mm}$ diameter), ions were focused by an Einzel lens into the pulser region of the TOF mass analyzer. The TOF analyzer chamber was pumped by two Leybold (Export, PA) model Turbovac 361350 1/s turbomolecular pumps. Deflection plates shield the low-energy ion beam from the high voltages applied to the accelerator column, and also make a final adjustment of the beam. Initially, the electric field in the gap of the pulser region is close to zero. The last grid of the extraction plates is held at a small positive potential (typically $6-12 \mathrm{~V}$ ) to prevent field penetration [11] from the accelerator column into the pulser region. When the pulser region is filled with ions, both push-out and draw-out voltage pulses are applied simultaneously to the extraction plates. The intermediate plate is kept at ground potential. The extraction pulses, produced by Behlke (Kronberg, Germany) model HTS 31GSM high voltage pushpull switches, have typical amplitudes of about $330 \mathrm{~V}$, a rise time of $30 \mathrm{~ns}$, and duration of $3 \mu \mathrm{s}$. The duration of the pulse is chosen to guarantee that the heaviest ions have enough time to leave the pulser region. The values of the draw-out and push-out pulses are chosen to ensure a homogeneous electric field between extraction plates. This reduces ion scattering near the intermediate grid where defocusing effects could be strong for ions with a relatively low kinetic energy. After leaving the pulser region, ions are accelerated to energies of about $5 \mathrm{keV}$ by a uniform DC field in the acceleration column. The ions then move with constant velocities in the field free drift region. The lengths of the pulser, acceleration and field free regions are $0.9 \mathrm{~cm}, 4.6 \mathrm{~cm}$ and $48 \mathrm{~cm}$, respectively. To keep the ion source and ion pulser potentials close to the ground, the drift region is floated at a high voltage potential. A perforated metal cover prevents penetration of the ground potential of the vacuum chamber into the drift region, while allowing 
effective pumping of the inside volume. Having traversed the drift region, ions enter a single-stage electrostatic mirror, which was tuned together with a dualstage accelerator to provide second-order space focusing on initial ion position [5]. The field free region is separated from the accelerator column and electrostatic mirror by BuckbeeMears (St. Paul, MN) grids with 114 wires/inch having a transmission of $88.6 \%$.

Reflected ions pass back through the drift region before striking a detector. The detector was assembled using a Hamamatsu (Bridgewater, NJ) resistance matched pair of microchannel plates with $12 \mu \mathrm{m}$ channel diameter and $12^{\circ}$ bias angle. The front plate of the detector was at the same potential as the drift region $(-5 \mathrm{kV})$, thus having the anode at high potential. The data acquisition system was decoupled from high voltage by ferrite transformer. The signal was amplified by an ORTEC (Oak Ridge, TN) model 9327 1-GHz amplifier and recorded by a 100 ps time digitizer (ORTEC model 9353). Voltages for the pulsers, drift region, and ion mirror were provided by Applied Kilovolts (Sussex, UK) models HP10 and HP5 stable, low noise, high voltage power supplies.

\section{Numerical Simulation}

Ion trajectory simulation was performed using SIMION 3D software (SIMION 7.0, ID National Engineering Laboratory, ID Falls, ID). The three-dimensional potential array had a cylindrical symmetry and used $2.2 \times 10^{6}$ grid points with $0.2 \mathrm{~mm}$ grid spacing for the simulation. Inscribed quadrupole diameter $d_{0}$ was determined by the relation: $d_{0}=d / 1.148$, where $d$ is the rod diameter [8]. The RF voltage of amplitude $200 \mathrm{~V}_{0-p}$, and frequency $\Omega / 2 \pi=1.0 \mathrm{MHz}$ was applied with $180^{\circ}$ phase difference between adjacent quadrupole rods to the first 25 $\mathrm{mm}$ long section. The radiofrequency voltages applied to the short quadrupole sections were either the same or were stepwise reduced toward the ion guide exit. The DC offset voltages were applied in a similar manner. The SIMION potential arrays were updated at time intervals of $0.05 \mu \mathrm{s}$. After passing the exit aperture, the ion beam was focused by an Einzel lens. The Einzel lens in the simulation included three cylindrical 4-mm thick electrodes with 4-mm diameter central holes located 2 $\mathrm{mm}$ apart from each other. The side electrodes were set to $0 \mathrm{~V}$ while the voltage on the central electrode of the Einzel lens was adjusted.

Seventeen ions with $m / z=500$ Th and initial energy of $0.03 \mathrm{eV}$ were chosen to simulate the ion beam passing through the segmented quadrupole. This initial energy corresponds approximately to the thermal energy at room-temperature and implies that ions were thermalized in collisions with buffer gas molecules before entering the segmented quadrupole ion guide simulated here. The entering ion's initial velocity vectors were evenly spread in the $80^{\circ}$ to $-80^{\circ}$ range relative to the axis of the ion guide. All ions started motion at the segmented quadrupole ion guide entry $(x=0, y=0)$.
Since the pressure in the segmented quadrupole was low (of the order of $10^{-3}$ torr), simulation didn't include collisions of ions with buffer gas molecules.

\section{Chemicals}

Solutions of peptides were prepared in molecular biology grade water, reagent grade methanol, and glacial acetic acid ( $2 \%$ in $1 / 1$ water/methanol). Bradykinin and Bombesin were purchased from Sigma-Aldrich (St. Lois, MO) and were used without further purification.

\section{Results and Discussion}

\section{Calculations}

To determine the divergence of the ion beam, which is defined as an angle deviation $\alpha$ in ion trajectories from the axis in a parallel beam, the voltage on the central electrode of the Einzel lens was adjusted to focus the ion beam exiting the segmented quadrupole. The divergence angle of the ion beam, exiting the quadrupole ion guide, can be determined by the following formula (in radians):

$$
\alpha=d_{\text {waist }} / F
$$

where $d_{\text {waist }}$ is the diameter of the beam waist at the point of beam's focus, and $F$ is the focal distance of the ion beam focus. Thus, the waist diameter $d_{\text {waist }}$ can be used as a measure of the beam divergence if the focal distance remains the same.

The results of numerical simulation of ion trajectories, in the case when the same RF and DC potentials are applied to all segments, are shown in Figure 3. Figure 3 a provides electric potential distribution. DC offset voltage and RF voltage amplitude were set to $20 \mathrm{~V}$ and $200 \mathrm{~V}_{0-p}$, respectively. The potential on the central electrode of the Einzel lens was set to 13.3 $\mathrm{V}$ to focus the ion beam at $F=21 \mathrm{~mm}$. The simulated ion beam waist diameter $d_{\text {waist }}$ was shown to be 0.40 $\mathrm{mm}$ (see Figure $3 \mathrm{~b}$ and its inset). When RF potentials were the same on all segments and DC potentials were reduced to $0 \mathrm{~V}$ in a stepwise manner, the ion beam divergence increased. The simulated beam waist diameter is shown to be $0.44 \mathrm{~mm}$. Figure 4 shows the results of numerical simulation with the RF voltage amplitudes decreased in a stepwise manner from $200 \mathrm{~V}_{0-p}$ on the first quadrupole section to $0 \mathrm{~V}_{0-p}$ on the last section, while the DC voltages are the same on all quadrupole sections. The potential on the central electrode of the Einzel lens was set to $13.4 \mathrm{~V}$ to focus the ion beam at the same plane as in the previous case. In the configuration shown in Figure $4 a$, the simulated ion beam waist diameter approximately equals $0.27 \mathrm{~mm}$ (see Figure $4 \mathrm{~b}$ and its inset). The largest improvement in ion beam divergence at the exit of the segmented quadrupole ion guide was 
A

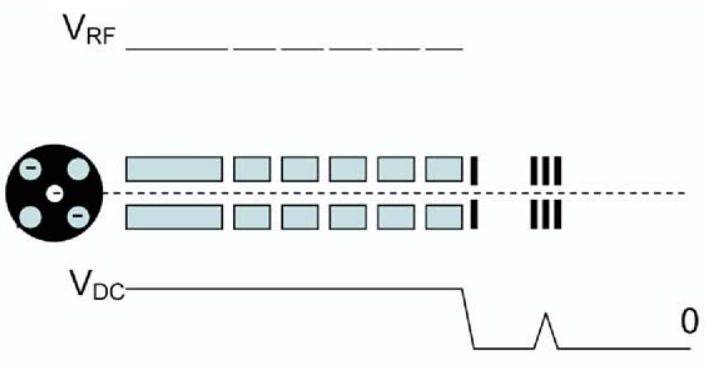

B

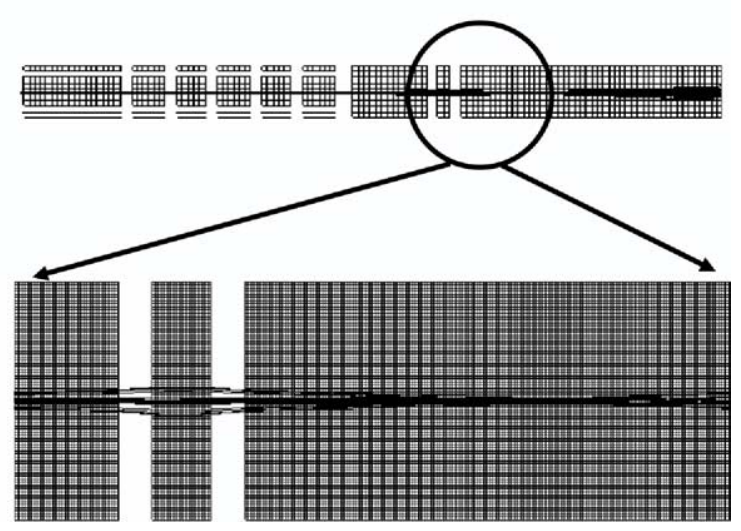

Figure 3. The results of numerical simulation of ion trajectories with constant RF and DC voltages.

obtained when both, RF and DC, voltages were reduced in a stepwise manner towards the exit of the ion guide. The results of numerical simulation of ion trajectories in this case are shown in Figure 5. The potential on the central electrode of the Einzel lens was set to $12.1 \mathrm{~V}$ to focus ion beam at the distance $F$

A

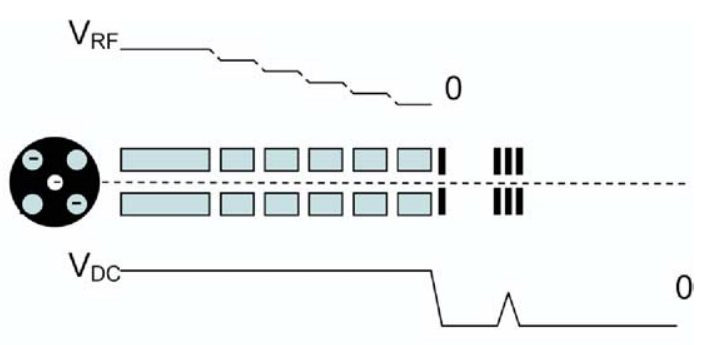

B

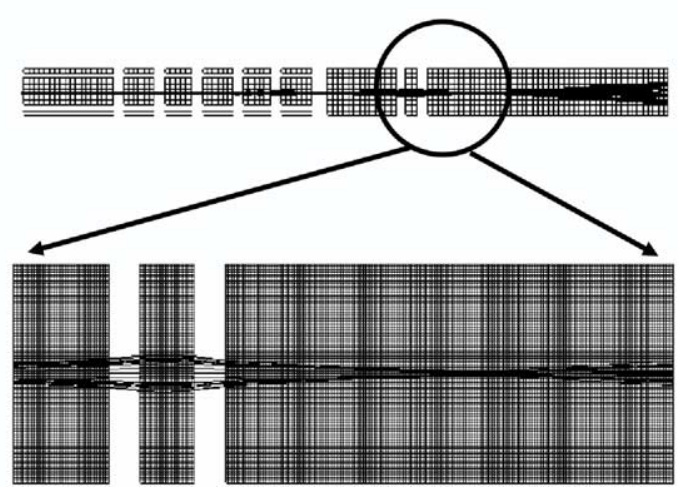

Figure 4. The results of numerical simulation of ion trajectories with constant DC and reducing RF voltages
A

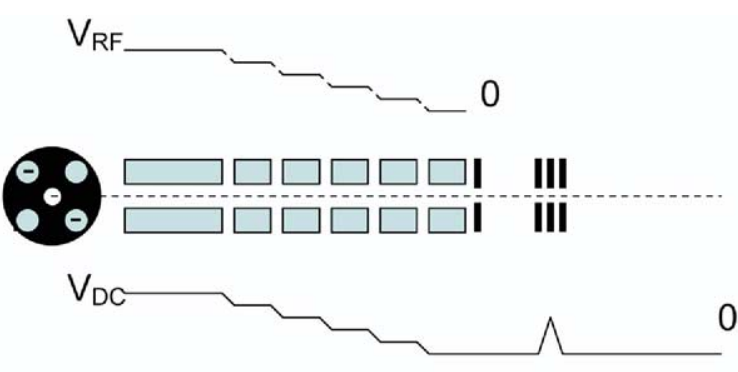

B

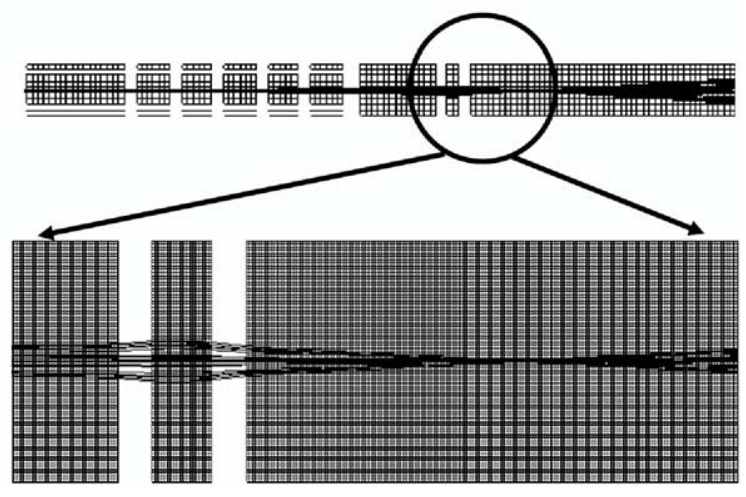

Figure 5. The results of numerical simulation of ion trajectories with reducing $\mathrm{RF}$ and $\mathrm{DC}$ voltages

$=21 \mathrm{~mm}$. As one can see on the inset of Figure $5 \mathrm{~b}$ $d_{\text {waist }}$ approximately equals $0.13 \mathrm{~mm}$.

\section{Experimental Results}

The electrospray spectra of peptide solutions were acquired on the oa-TOF instrument described above. The concentration of peptides was $1 \mu \mathrm{M}$, flow rate -1 $\mu \mathrm{l} / \mathrm{min}$, and ESI voltage $-2.2 \mathrm{kV}$. The spectra were acquired for $10 \mathrm{~s}$ with a TOF repetition rate of $5 \mathrm{kHz}$. Several different RF and DC voltage distributions on the segmented quadrupole ion guide were tested.

The isotopic structure of the Bradykinin (2+) ion is shown in Figure 6. The voltage distributions on the last segmented quadrupole ion guide were changed by connecting $\mathrm{RF}_{\text {out }}$ and $\mathrm{DC}_{\text {out }}$ on the electrical feedthroughs either to the voltage source, or to the ground. In case $1, \mathrm{RF}_{\text {out }}$ was connected to the output of the $\mathrm{RF}$ transformer and $\mathrm{DC}_{\text {out }}$ was connected to the $\mathrm{DC}$ power supply. In case $2, \mathrm{RF}_{\text {out }}$ was grounded and $\mathrm{DC}_{\text {out }}$ was connected to the $\mathrm{DC}$ power supply. In case $3, \mathrm{RF}_{\text {out }}$ and $\mathrm{DC}_{\text {out }}$ were grounded. Switching was performed outside the vacuum, simply by connecting the corresponding cables to different outputs, thus providing almost instantaneous comparison. As one can see from the spectra presented on Figure 6, the mass resolution (determined as $m / \Delta m$ at FWHM) improves considerably when the RF and DC voltages applied to the quadrupole guide sections were reduced towards the exit.

Similar results were demonstrated for the Bombesin $(3+)$ ion (see Figure 7). The experimental conditions were analogous to that referred to Figure 6. To quanti- 


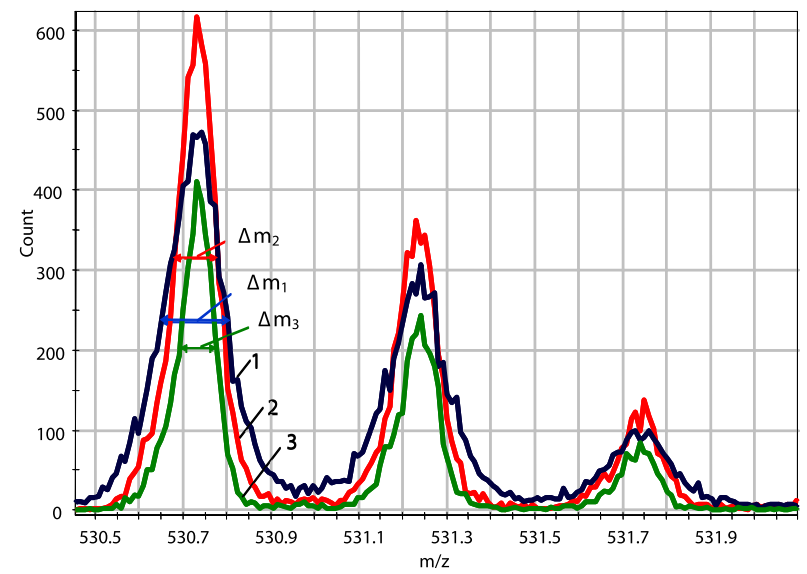

Figure 6. Isotopic structure of Bradykinin (2+) ion; 1-corresponds to the same rf and DC voltages applied to quadrupole segments, 2-DC voltages are the same and RF voltages are stepwise reduced towards the ion guide exit, 3-both, RF, and DC, voltages are stepwise reduced towards the ion guide exit.

tate the mass resolution changes during experimental measurements, the data on mass resolution were averaged over 15-20 spectra acquired under identical conditions:

\begin{tabular}{lcc}
\hline & Bradykinin (2+) & Bombesin (3+) \\
\hline \hline Case 1 & $3470 \pm 110$ & $3480 \pm 170$ \\
Case 2 & $4820 \pm 140$ & $4770 \pm 140$ \\
Case 3 & $5320 \pm 170$ & $5410 \pm 140$ \\
\hline
\end{tabular}

Similar improvements in resolution were observed over a whole mass range of electrospray generated peptide ions, for example, for Bradykinin $(3+)(\mathrm{m} / \mathrm{z}=$ 354.19) and Bradykinin $y_{7}(1+)$ fragment $(m / z=807.42)$

Ion motion within the quadrupole ion guide in the radial direction is determined by the trapping RF potential which in a pseudo-potential approximation can be determined as [8]

$$
U_{\text {trap }}=q V_{R F} / 4
$$

where

$$
q=4 V_{R F} z e / m \Omega^{2} r_{0}^{2}
$$

is a dimensionless Mathieu parameter describing the stability of the ion's radial motion; $\Omega=2 \pi f$ is a circular frequency of the RF voltage, $V_{R F}$ is its amplitude (zero-to-peak and pole to ground), and $r_{0}$ is an inscribed quadrupole radius. In the pseudo-potential well approximation (which is normally valid at $q<0.4$ ), the ion motion can be described as a harmonic secular oscillation with a frequency $\omega \ll \Omega$ within a parabolic potential well having the depth of $U_{\text {trap }}$ with much smaller "ripple" oscillations at the drive frequency $\Omega$ [8].

Ions moving along the ion guide typically fill a

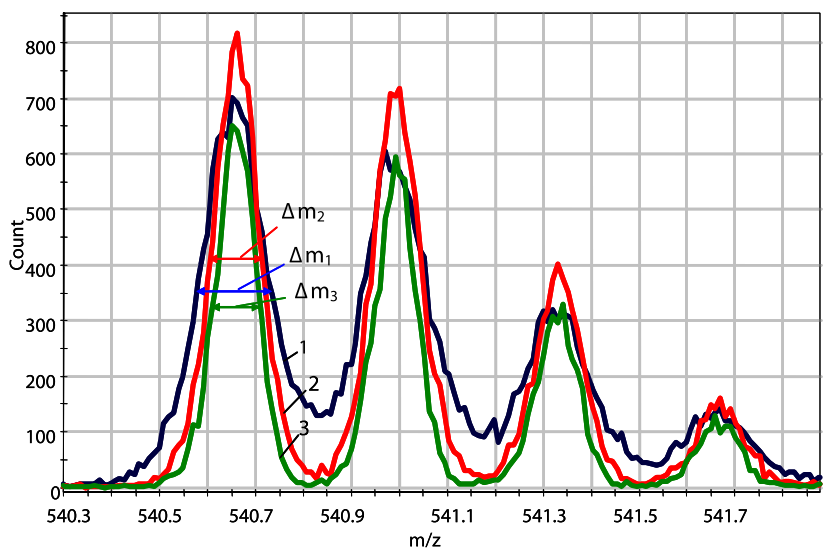

Figure 7. Isotopic structure of Bombesin $(3+)$ ion; $1-$ corresponds to the same RF and DC voltages applied to quadrupole segments, 2-DC voltages are the same and RF voltages are stepwise reduced towards the ion guide exit, 3-both, RF, and DC, voltages are stepwise reduced towards the ion guide exit.

central area near the ion guide axis which is determined by the pseudo-potential well depth. The average kinetic energy in the radial direction, after thermalization via collisions with buffer gas molecules, usually is close to room-temperature (i.e., in a vicinity of $295 \mathrm{~K}$ or $0.03 \mathrm{eV}$ ). When ions leave the ion guide, they experience the radial kick-off effect, which is due to the cut-off of the $\mathrm{RF}$ ion oscillations at the arbitrary phase. The RF potential distribution between the ion guide exit and the exit aperture can be described as [12]:

$$
\Phi=V_{R F} \cos \Omega t \frac{x^{2}-y^{2}}{r_{0}^{2}} f(z)
$$

where $f(z)=1-\exp \left[-a(1-z) / r_{0}-b(1-z)^{2} / r_{0}^{2}\right]$ is a fast decreasing ( $a=2.13$ and $b=1.55$ ) exponential function [13] in units of $(1-z) / r_{0} ; z$ is the coordinate along the quadrupole axis originating at the quadrupole exit; 1 is the distance between the rods and exit aperture. The force acting on the ion in this region in the y direction is determined as:

$$
F_{y}=-z e \frac{\partial \Phi}{\partial y}=2 z e V_{R F} \cos \Omega t \frac{\mathrm{y}}{r_{0}^{2}} f(\mathrm{z})
$$

where $y$ is linked with a slowly varying secular motion coordinate $\mathrm{Y}$ by the relation $\mathrm{y}=\mathrm{Y}-0.5 q \mathrm{Y} \cos \Omega t$ in the pseudo-potential well approximation [8]. The resulting velocity gain in the y direction when the ion leaves the ion guide equals to:

$$
\Delta \mathrm{v}_{\mathrm{y}}=\int_{t_{0}}^{t_{f}} 2 z e V_{R F} \cos \Omega t \frac{\mathrm{Y}-0.5 \mathrm{q} Y \cos \Omega t}{m r_{0}^{2}} f(\mathrm{z}) d t
$$

where $t_{0}$ is the time when the ion leaves the ion guide and $t_{f}$ - the time when the ion reaches the exit aperture. Since $f(z)$ is decreasing exponentially, only a small area 
near the quadrupole exit will contribute significantly to the integral (7). Hence, we can assume that $\cos \Omega t$ and $Y(t)$ don't change significantly in this area, which gives an estimate of the value of the integral (7) as $2 z e V_{R F}$ $\cos \Omega t_{0} \mathrm{Y}\left(t_{0}\right) / m r_{0}^{2 *} 1 / \mathrm{v}_{\mathrm{z}}$. As one can see, the radial velocity gain is especially significant for the ions moving off the axis (larger $\left.Y\left(t_{0}\right)\right)$. The value of the velocity gain is directly proportional to the strength of the RF field at the ion guide exit and inversely proportional to the ions velocity in the axial direction, (which reduces the time spent in the fringing field).

In approximation of a negligible space charge and equilibrium of the ion's kinetic energy with buffer gas, the ion density distribution can be described by the Boltzmann distribution for ions moving in the potential well formed by the effective potential [14]. The averagesquared radial position $R_{T}$ is as follows [14]: $R_{T}=2 r_{0}$ $\left(k T / z e q V_{R F}\right)^{1 / 2}$. For Bradykinin (2+) ions, $T=295 \mathrm{~K}, r_{0}=$ $3.48 \mathrm{~mm}, V_{R F}=200 \mathrm{~V}, f=1 \mathrm{MHz}$ one can obtain $q=0.3$ (eq 4) and $R_{T}=0.11 \mathrm{~mm}$. Using this value as average for $\mathrm{Y}\left(t_{0}\right)$ and $2 / \pi$ as average for $\cos \Omega t_{0}$, together with $1=1.5$ $\mathrm{mm}, \mathrm{v}_{\mathrm{z}}=2.6 \times 10^{3} \mathrm{~m} / \mathrm{s}$ (this velocity corresponds to 20 $\mathrm{eV})$, one can estimate average $\Delta \mathrm{v}_{\mathrm{y}}$ as $200 \mathrm{~m} / \mathrm{s}$. For the value of $E=6 \times 10^{4} \mathrm{~V} / \mathrm{m}$, typical for our experimental conditions, this gives the value of turn-around time from eq 1, 37 ns. This would limit the resolution in the TOF mass spectrometer to $\sim 500$. Since the experimental value is higher, this means that the ion beam is substantially clipped by the exit ion optics apertures before the ions enter the pulser region. In the experiments described here the mass resolution resulting from this limited ion beam is compared for two cases of constant and gradually changed RF and DC voltage distributions along the quadrupoles near the ion guide exit.

\section{Conclusions}

The velocity spread in the direction orthogonal to the ion guide axis, caused by fringing fields at the ion guide exit, increases the turn-around time and decreases the mass resolution of the TOF mass analyzer. The proposed design of the ion guide effectively minimizes this effect by reducing the strength of the RF field and simultaneously increasing the axial ion velocity at the exit. Decreasing ion beam divergence resulted in considerably improved mass resolution of the studied instrument.

\section{References}

1. Gerlich, D. In State-Selected and State-to-State Ion-Molecule Reaction Dynamics, Part 1: Experiment. Vol. LXXXII; Ng, C. Y.; Baer, M., Eds.; John Wiley: New York, 1992, pp 1-176.

2. Douglas, D. J.; French, J. B. Collisional Focusing Effects in Radio Frequency Quadrupoles. J. Am. Soc. Mass Spectrom. 1992, 3, 398-408.

3. Stafford, G. C.; Kelley, P. E.; Syka, J. E. P.; Reynolds, W. E.; Todd, J. F. J. Recent Improvements in and Analytical Applications of Advanced Ion Trap Technology. Int. J. Mass Spectrom. Ion Processes 1984, 60, 85-98.

4. Krutchinsky, A. N.; Chernushevich, I. V.; Spicer, V. L.; Ens, W.; Standing, K. G. Collisional Damping Interface for an Electrospray Ionization Time-of-Flight Mass Spectrometer. J. Am. Soc. Mass Spectrom. 1998, 9, 569-579.

5. Mamyrin, B. A.; Karataev, V. I.; Shmikk, D. V.; Zagulin, V. A. Sov. Phys. JETP 1973, 37, 45-53

6. Chernushevich, I. V.; Loboda, A. V.; Thomson, B. A. An Introduction to Quadrupole-Time-of-Flight Mass Spectrometry. J. Mass Spectrom. 2001, 36, 849-865.

7. Wiley, W. C.; McLaren, I. H. Time-of-Flight Mass Spectrometer with Improved Resolution. Rev. Sci. Instrum. 1955, 26, 1150-1157.

8. Dawson, P. H. Quadrupole Mass Spectrometry and Its Applications; Elsevier: Amsterdam, 1976.

9. Fite, W. L. Spatial Separation of Fringe Fields in Quadrupole Mass Filters. Rev. Sci. Instrum. 1976, 47, 326-330.

10. Jones, R. M.; Gerlich, D.; Anderson, S. L. Simple Radio-Frequency Power Source for Ion Guides and Ion Traps. Rev. Sci. Instrum. 1997, 68, 3357-3362.

11. Dodonov, A. F.; Chernushevich, I. V.; Laiko, V. V. Electrospray Ionization on a Reflecting Time-of-Flight Mass Spectrometer. In Time-of-Flight Mass Spectrometry; Cotter, R. J., Ed.; ACS: Washington DC; 1994; pp 108-123.

12. Londry, F. A.; Hager, J. W. Mass Selective Axial Ion Ejection from a Linear Quadrupole Ion Trap. J. Am. Soc. Mass Spectrom. 2003, 14, 1130-1147.

13. Hunter, K. L.; McIntosh, B. J. An Improved Model of the Fringing Fields of a Quadrupole Mass Filter. Int. J. Mass Spectrom. Ion Proc. 1989, 87, 157-164.

14. Tolmachev, A. V.; Udseth, H. R.; Smith, R.D. Modeling the Ion Density Distribution in Collisional Cooling RF Multipole Ion Guides. Int. J. Mass Spectrom. 2003, 222, 155-174. 